\title{
Gestionando la vulnerabilidad desde los cuidados comunitarios en la política local: la experiencia de Zumarraga
}

\section{Patricia Martínez García}

Aradia Cooperativa

patmartinez.garcia@gmail.com

\section{Delicia Aguado-Peláez}

Aradia Cooperativa

deliciaaguado@gmail.com
Hainbat euskal hiritako testuinguru politiko eta soziala paradigma industrialaren higaduratik eratorritako erronka berriek markatzen dute. Krisi ekonomikoaren aurrean harturiko neurriek kolektibo ezberdinek bizi duten kalteberatasun egoera eta hauen arteko ezberdintasunak areagotu ditu. Erakunde publikoek, kasu guztietan horrela ez bada ere, gizabanako eta kolektibo babesgabeen arteko lehia-logiketan oinarrituriko politika publikoen alde egin dute. Horrela, artikulu honen helburua, aipaturiko erronken aurrean komunitate-loturak indartzea sustatzen duten estrategia lokalak bistaratzea da. Honetarako, Zumarragako udalerria hartuko da ikergai moduan, bertan ekintza politiko eta sozialaren erdigunean zaintza kokatzen duten ekimenak gauzatzen ari baitira.

\section{GAKO-HITZAK:}

Zaintza, komunitatearen garapena, intersekzionalitatea, toki-politika, Zumarraga.
El contexto político y social de muchas ciudades vascas está marcado por nuevos retos que derivan del desgaste del paradigma industrial. Las respuestas a la crisis económica han provocado el aumento de las desigualdades y de las situaciones de vulnerabilidad de muchos colectivos. Desde las instituciones se ha optado por políticas públicas sustentadas en el reforzamiento del individualismo y de las lógicas de competitividad entre grupos desfavorecidos, pero no siempre. El objetivo de este artículo es visibilizar estrategias locales que buscan robustecer los lazos comunitarios como salida a los desafíos mencionados. Para ello, se utiliza como caso de estudio el municipio de Zumarraga, donde se están desarrollando iniciativas que ponen los cuidados en el centro de la acción política y social.

\section{Palabras Clave:}

Cuidados, desarrollo comunitario, interseccionalidad, política local, Zumarraga. 


\section{Introducción}

El paradigma fordista desde el que muchas ciudades crecieron se está desgastando. El debilitamiento de las fábricas, el envejecimiento poblacional y un fuerte individualismo son algunos de sus expresiones más visibles. Unos síntomas que la teoría y la praxis feminista han venido denunciando como crisis multidimensional que afecta a la economía entendida desde su origen etimológico-oikonomos-, que alude a la administración -nemein-del hogar -oikos- (Pérez Orozco, 2011). Y, con ello, la ecología, la sociedad y los cuidados quedan relegados a un segundo plano en un sistema que prioriza la producción sobre todas las cosas, imponiendo el imperio del conocido como homo economicus (Mellor, 2007; Pérez Orozco, 2014; Marçal, 2017; Juliano, 2017).

En este sentido, nuestras sociedades están insertas en una matriz de dominación, utilizando la noción de Patricia Hill Collins (1990), atravesada por lógicas capitalistas, patriarcales y coloniales, a las que se pueden sumar las edadistas y capacitistas, entre muchas otras. Esto deviene en una estructura que excluye a todos aquellos sujetos que no encajan en lo que se ha definido como normativo. En este sentido, Mary Mellor explica (2007: 42):

La economía solo quiere al "hombre" cuando este está en forma, maduro, pero no viejo, capaz de moverse y sin demandas extrañas. La economía no necesita su infancia, sus enfermedades, su hambre, su necesidad de descanso y sueño, sus prendas sucias, sus preocupaciones, el cuidado de sus hijos, sus envejecimientos, sus responsabilidades. Obviamente, en estos casos el hombre también puede ser mujer [el género del hombre económico no es una división biológica absoluta].

Esta estructura de dominación no es una ficción -tampoco una exageración- sino que es la base de nuestra organización económica, política y social que se refleja en las injusticias en el ámbito de la distribución, el reconocimiento y la representación (Fraser, 2008; 2015). Es decir, las mujeres -y otros colectivos subalternos- están discriminadas en lo económico, en lo simbólico y en el acceso a la toma de decisiones. Por poner algunos ejemplos que han ganado protagonismo en los últimos tiempos por las reivindicaciones feministas: la precariedad y marginalidad de los empleos feminizados o la invisibilidad de los trabajos de cuidados que tienen consecuencias reales en las experiencias de pobreza de muchas mujeres mayores con bajas pensiones 0 en la explotación de muchas trabajadoras del hogar, de hoteles o de residencias.

La constante de estas situaciones es, como se ha mencionado, una estructura sustentada en la fantasía de individualidad, parafraseando el título de Almudena Hernando (2012), en la que solo se valoran aquellas cuestiones que tiene un valor en el mercado, mientras son las actividades no monetizadas las que sostienen la vida, individual y colectiva, sea el trabajo doméstico, el voluntario o la participación ciudadana (Orozco, 2006; Marçal, 2017). En consecuencia, se niega la ecodependencia e interdepedencia de la existencia humana o, en otras palabras (Herrero, Pascual y González Reyes, 2018: 14-16):

La vida humana se desarrolla inserta en un medio natural del que forma parte [...]. Como todas las especies vivas, para existir y reproducirnos dependemos de una naturaleza que nos proporciona todo lo que necesitamos para vivir [agua, oxígeno, alimentos...]. Pero, además, los seres humanos tenemos una segunda dependencia material que viene dada por el hecho de que nuestra vida transcurra encarnada en cuerpos que nacen, enferman, envejecen y tienen necesidades diferentes. Nuestros cuerpos solo pueden sobrevivir si se insertan en un espacio de relaciones que garantice cuidados y atenciones a lo largo de toda la vida, y sobre todo en algunos momentos especialmente vulnerables del ciclo vital.

El reconocimiento de la noción que recoge esta cita supone alterar todo el entramado sobre el que se han materializado las prácticas normativas e institucionales que, como se ha dicho, responden a un modelo productivista todavía centrado en la figura del ganador de pan y que dificulta las trayectorias vitales. A este respecto, las instituciones en los distintos niveles territoriales, impregnadas de una ideología neoliberal, han optado por amparar los privilegios, agravando las situaciones de vulnerabilidad.

Sin embargo, en muchos casos, el contexto local ha sido un ámbito de resistencia desde el que se ha optado por iniciativas de inclusión y fortalecimiento comunitario (Collins, 2017; Navarro Pedreño, 2017; Villasante, 2017). Aunque incipiente, este es el camino elegido por la experiencia en la que se basa este artículo: procesos sustentados en un modelo de cuidados por parte de Zumarraga. Este municipio del interior de Gipuzkoa, afectado por el debilitamiento de un sector industrial en el que había sido referente, se convierte en un espacio estratégico desde el que (re)pensar la acción política y social local hacia la generación de alternativas que superen el paradigma tradicional.

\subsection{Hacia lógicas sustentadas en los cuidados (comunitarios)}

En los últimos años, especialmente al amparo de las jornadas del 8 de marzo de 2018 y 2019, los cuidados se han puesto en el centro de las reivindicaciones feministas para transformar el modelo social. Cuando hablamos de cuidados hacemos referencia a todas aquellas actividades imprescindibles para hacer que "la vida sea vivible" (Juliano, 2017: 96) en una dimensión material pero también afectiva y relacional 
(Carrasquer, 2013). Esto abarca todas las tareas destinadas a proporcionar bienestar emocional, físico y psíquico, con el fin de cubrir las necesidades diversas de todas las personas y sus diferentes etapas (Comas, 2014).

Este bienestar suele ser generado desde los recursos públicos y privados, junto con la red de personas allegadas, pero para el que solemos olvidarnos de una fuente de provisión fundamental: la comunidad (Collins, 2017; Arrieta et al., 2018). Así, un modelo sustentado en los cuidados comunitarios habla de una alternativa transformadora en el que la ciudadanía se une para generar soluciones conjuntas a las distintas vulnerabilidades presentes en la sociedad, asumiendo la (inter)dependencia de las personas.

En un diálogo entre la noción de cuidadanía ${ }^{1}$ y desarrollo comunitario, se destacan cuatro grandes principios: conectar, conocer, compartir y construir. Es decir, activar los lazos entre la ciudadanía, especialmente entre aquellos colectivos en mayor situación de vulnerabilidad, más proclives a sufrir "dolores sociales"; acercar a las personas para desmontar estereotipos y prejuicios y buscar puntos en común; colaborar en la generación de definiciones comunes sobre el qué queremos, e ir colocando los cimientos del nuevo modelo. Zumarraga está incorporando, lenta y progresivamente, estas lógicas a su acción local. Una apuesta a largo plazo, ya que la creación de espacios de conocimiento mutuo y confianza, donde las personas, desde su diversidad, se miren, se entiendan y se hablen sin jerarquías buscando "los puntos de anclaje común que nos aproximan unos a otros" (Medina, 2005: 19). Procesos humildes, largos, tomados con serenidad y tiempo porque "no hay empatía apresurada" (Medina, 2005: 24), desde los que experimentar con estas sinergias.

\section{Metodología}

Con el fin de visibilizar estrategias locales que se encaminan hacia fórmulas alejadas de la otredad y la exclusión en las respuestas a los efectos devenidos de la crisis, este artículo parte de la experiencia en Zumarraga y de su preocupación por incorporar lógicas participativas e inclusivas en acción política. A este objetivo responde el proyecto "Construyendo propuestas hacia un modelo social sustentado en los cuidados" (2018) ${ }^{2}$, cuyos resultados se van a

${ }^{1}$ En 2004, Carolina Junco, Amaia Pérez Orozco y Sira del Río escriben un manifiesto en el que definen la cuidadanía como "una nueva forma de reconocernos como sujetos responsables de una sociedad no jerárquica que apueste por poner la vida en el centro. Entendiendo la cuidadanía como un proceso vivo que va a ir destapando y relacionando diferentes realidades. Apareciendo protagonistas que se suelen mantener ocultos/as a lo social y lo político. Abriéndonos a la diversidad y cooperación entre distintas luchas, siempre apostando por poner la vida y su mantenimiento en el centro".

${ }^{2}$ Este proyecto continúa con el trabajo elaborado por Igor Ahedo, Unai Aranda y Miriam Ureta, del grupo de investigación consolidado Parte Hartuz (UPV/EHU): “Diagnóstico de la Diversidad Social y Vul- utilizar en el presente texto. Para ello, se parte de las herramientas brindadas por la investigaciónacción participativa (IAP), técnicas que nos permiten analizar la realidad desde una reflexión que parte e implica a las personas protagonistas, (re)valorizando sus saberes y experiencias desde un enfoque de horizontalidad.

Partiendo de que las alternativas participativas no son siempre inclusivas (Martínez Palacios y MartínezGarcía, 2017), se incorpora la interseccionalidad como herramienta imprescindible para la experimentación social (Collins, 1990; Crenshaw, 1991). Porque este enfoque no solo nos permite analizar la complejidad de la maraña de ejes que nos atraviesan -como clase cultural y clase económica, edad, género, origen, racialización, situación de dependencia, situación familiar...-, sino también a visibilizar todas esas prácticas que surgen en los colectivos de las periferias y que contribuyen a imaginar otras formas de sentir, estar y construir la ciudad desde los cuidados comunitarios.

Desde estos marcos de referencia, se desarrollaron espacios de reflexión individual, a través de veinticuatro entrevistas en profundidad, realizadas a diferentes agentes políticos y técnicos, así como a ciudadanía organizada y no organizada. También se realizaron encuentros para la reflexión grupal mediante dos talleres en los que se incorporaron un total de diecisiete personas, en los que también se busca una capacitación en cuidados y desarrollo comunitario.

Toda la información obtenida posibilita profundizar en la identificación de necesidades de la población $y$, especialmente, de colectivos en situación de vulnerabilidad, así como en propuestas para responder a dichas necesidades. Es decir, se centra en ese cambio de paradigma que ponga los cuidados y la comunidad en el centro para responder a los retos - presentes y futuros- que se plantean en el municipio.

\section{Una oportunidad para el cambio social}

Zumarraga comparte destino con otros muchos municipios que crecieron al amparo de la industria. Lugar de acogida de inmigrantes interiores procedentes del Estado español en la década de los sesenta, constituye un escenario peculiar en Gipuzkoa tanto a nivel político como social y cultural (Prada, 2016). El acelerado crecimiento de la población -junto con su orografía- influye en la propia configuración del municipio y condiciona el desarrollo social de quienes lo habitan, ya que los barrios se convierten en vertebradores de muchas de

nerabilidad en Zumarraga" (2017/2018), para el Área de Servicios Sociales, Igualdad y Diversidad del ayuntamiento, y de otras iniciativas diagnósticas y experimentales elaboradas para el Área de Administración Local y Participación Ciudadana, también por Igor Ahedo, así como por la cooperativa Aradia. 
las vulnerabilidades actuales, con zonas altamente envejecidas o donde se concentran personas con menor capacidad económica o racializadas.

La distribución de los barrios también es un indicador de las tensiones que se experimentaron entre las familias obreras del Estado español y las familias autóctonas, que se han ido superando con las generaciones sucesivas. No obstante, el progresivo desmantelamiento de la industria ha contribuido a la aparición, muy incipiente, de discursos excluyentes respecto a otra migración procedente de países ajenos a la Unión Europea, especialmente de América del Sur y Central, de Marruecos y Pakistán. Aunque tanto Gipuzkoa como el municipio cuentan con unos niveles de bienestar elevados, tras la crisis económica y el adelgazamiento del Estado de Bienestar, surgen expresiones de lo que se ha llamado guerra entre pobres (Ahedo et al., 2018). Es decir, van apareciendo los primeros discursos de desconfianza hacia colectivos vulnerables, que se empiezan a percibir como competencia a la hora de conseguir un empleo, pero también a la hora de optar por ayudas sociales. Una desconfianza que está construida sobre estereotipos y prejuicios y que afecta, especialmente, a una parte de la población que está ligada con ejes como clase social, origen o colectivos altamente estigmatizados.

En definitiva, el fuerte impacto de la crisis en Zumarraga no solo ha sido económico y social, sino que también ha afectado al propio imaginario de sus habitantes, debilitándose la idea de "Zumarraga industrial y de acogida”. El desgaste de esta etiqueta conlleva la pérdida del pegamento que había funcionado en las últimas décadas, agudizando la desconexión estructural. Por ello, y ante las crecientes respuestas excluyentes en otras latitudes, la apuesta municipal pasa por el reforzamiento de los lazos comunitarios, atendiendo a una comprensión interseccional de la diversidad.

\subsection{Vulnerabilidades en la encrucijada interseccional}

La incorporación de un enfoque interseccional posibilita comprender la complejidad de quienes habitan Zumarraga y sus distintas formas de experimentar los dolores sociales, que se padecen de forma más o menos intensa según la clase, la diversidad funcional, la edad, el nivel de estudios, la diversidad funcional, el origen o estar o no racializada, entre otros marcadores. A este respecto, y teniendo siempre en cuenta el carácter dinámico de la experimentación de las opresiones y los privilegios (Collins, 1990), a continuación se recogen las encrucijadas que parecen jugar un papel relevante en la actualidad (Aguado-Peláez y Martínez García, 2018; Ahedo et al., 2018) y que, probablemente, se pueden extender a otros entornos territoriales similares.

En primer lugar, la clase y la racialización surgen como factores fundamentales en lo que se ha llamado la guerra entre pobres. Una referencia que aparece a menudo en las entrevistas individuales es la comunidad gitana, un grupo históricamente estigmatizado en esta localidad y en la gran mayoría de territorios del Estado español (Giménez, Cortés y Sáez, 2016). Los miembros de este grupo de clase humilde -especialmente quienes están en desempleo- se convierten en diana de desconfianza en algunos barrios, aunque parece que las tensiones se han ido debilitando con el paso de los años. Por ello, se ha señalado la importancia de generar estrategias para superar las barreras que limitan su acceso al empleo, a la vivienda, al reconocimiento como cultura local o la propia participación en la comunidad, incluso entre los más jóvenes.

Otro colectivo racializado y al que se aplican lógicas de otredad son las personas procedentes de países musulmanes. Esta comunidad es creciente y es fundamental reforzar los lazos sociales con una nueva generación de personas migradas, evitando discursos que fomenten la exclusión y los estereotipos. A este respecto, es importante señalar la labor realizada por colectivos como Mujeres Musulmanas Al Amal, que son un referente social y cultural. Asimismo, si bien los discursos se encaminan a otros estereotipos menos victimizantes y más ligados a la naturalización de su afectividad, las mujeres de países latinos también experimentan prácticas racistas. Todas las mujeres racializadas que han participado en este proceso, aunque expresan que Gipuzkoa y Zumarraga son lugares inclusivos en general, se sienten relegadas, entre otras cuestiones, en el mercado laboral.

En segundo lugar, otra entrecruzada relevante es la conformada por la clase, la edad y la familia. Aunque hay una dotación de recursos de calidad, ligado a un mayor desarrollo del bienestar en Euskadi y en el territorio histórico (Martínez-Buján, 2014), es un modelo todavía marcadamente familista. Así, el apoyo familiar -económico o psicológicose convierte en clave para la inclusión social. Algo que se observa más claramente en las clases humildes y en aquellas edades donde la recepción de cuidados es más acuciante, como en la infancia y la adolescencia. A este respecto, es fundamental destacar la existencia de un grupo de jóvenes en riesgo de exclusión sin ningún tipo de amparo institucional, cuya vulnerabilidad está muy agravada por la falta de salidas educativas y laborales, la ausencia de redes familiares y sociales y el origen ${ }^{3}$.

Al otro lado de la encrucijada se encuentran personas sin apoyo familiar y, especialmente, sin muchos recursos económicos, pero en esta ocasión de edad avanzada. Se trata de un problema creciente porque la sociedad zumarragatarra está muy envejecida, con

${ }^{3} \mathrm{~A}$ este respecto, es importante destacar como respuesta institucional-comunitaria el trabajo activo con niños y niñas menores de edad a través del programa Topaleku -ubicado en el barrio de Eitza-, señalado como un modelo exitoso para la inclusión social. No obstante, se señala como insuficiente ya que, al cumplir la mayoría de edad, las y los jóvenes se quedan sin apoyo al ser consideradas plenamente independientes y autónomas, algo que las deja en una alta situación de vulnerabilidad. 
una pirámide poblacional prácticamente en forma de hexágono, con bolsas importantes de precariedad entre quienes no tienen pensiones adecuadas. En este sentido, la soledad y la desconexión se señalan continuamente como un problema central. Además, se reivindica una diversificación de intereses y ser reconocidos como agentes activos en la comunidad (Fundación Matia, 2016). En todos estos cruces, estar en una situación de dependencia aparece como agravante de vulnerabilidad - ligada a la edad o a alguna discapacidad física, psíquica o enfermedades mentales-. De nuevo, es fundamental destacar como se señala la necesidad de más recursos públicos dedicados a personas de apoyo y acompañamiento y, especialmente, de profesionales.

Por último, el género aparece de forma transversal como un eje que agudiza todos los dolores sociales. En el rol de cuidadoras altruistas la carga de la crianza y del bienestar de las personas en situación de dependencia recae en las mujeres, naturalizando su adscripción al ámbito reproductivo no mercantilizado. En la extensión de este papel social en lo productivo, su presencia es casi exclusiva en las profesiones más precarias, como las empleadas del hogar y de cuidados - con un fuerte componente de vulnerabilidad cuando son migradas-, cuya marginalidad influye en su calidad de vida presente y futura ${ }^{4}$. También se presentan más desconectadas y afectadas por la soledad en dos grupos señalados como vulnerables: aquellas mujeres extranjeras, sin manejo del idioma, que están desvinculadas de la comunidad, y las mujeres de edad avanzada que están en situación de soledad no deseada.

No obstante, y volviendo al carácter complejo y dinámico de la opresión que nos ofrece la herramienta interseccional, estas vulnerabilidades se convierten en una oportunidad para desarrollar un paradigma alternativo para el municipio. En este sentido, las mujeres, en toda su diversidad, se entienden protagonistas de un modelo de cuidados comunitarios, y también son reconocidas como tales. Ya son muchas las experiencias asociativas puestas en marcha y que son percibidas como cercanas y claves para la conexión alrededor de la comunidad y la escuela. A este respecto, el encuentro establecido entre ellas en los distintos talleres refuerza esta percepción, siendo agentes clave en la extensión de las lógicas de diálogo y convivencia que han mantenido históricamente desde la invisibilidad.

En este sentido, el cambio de paradigma pasa por poner en valor las experiencias pasadas y presentes de mujeres que han ido tejiendo de manera invisible los lazos que hoy permiten hablar de comunidad y que tienen que ser reforzados. Como aboga Silvia Navarro Pedreño (2017: 25):

${ }_{4}^{4}$ Sobre esta cuestión se puede señalar el trabajo realizado por Patricia Martínez-García y Delicia Aguado-Peláez (2019) al amparo de la beca de investigación de la Diputación Foral de Gipuzkoa: “¿Quién sostiene la vida? Análisis interseccional de las cuidadoras remuneradas en Zumarraga y Urretxu".
Lo que durante mucho tiempo se ha considerado un saber y una experiencia de segundo orden, propio de lo femenino, alejados del paradigma racionalista y objetivista, levantado desde el dominio patriarcal y el señorío de lo masculino, hoy creo que es una valiosa fuente de oportunidad. Este saber es capaz de crear otros mundos posibles y renovadas alternativas de acción y de transformación, es decir, de construir la vida y lo social desde un territorio en el que entren en juego la subjetividad, las emociones, la creatividad, la belleza, la alteridad, el cuidado, la vitalidad, lo sensual, el cuerpo y el alma.

\subsection{Construyendo espacios de encuentro y activando la comunidad}

Aunque son muchas las potencialidades señaladas -muy ligadas a la existencia de recursos públicos y sociales- las personas participantes verbalizan una serie de necesidades que tienen que ver con el debilitamiento progresivo de la comunidad. Por un lado, se habla de la carencia de espacios de encuentro para conocerse y evitar muchos de los prejuicios que se proyectan sobre los colectivos en situación de vulnerabilidad. Esta carencia se ve agravada por un debilitamiento del tradicional tejido asociativo de carácter asistencial-social -aunque se mantienen y se refuerzan las vinculadas a la cultura vasca y las deportivas-, por una desconexión importante entre barrios y por el debilitamiento de la red vecinal. En este sentido, las desconfianzas entre institución local y ciudadanía y la carencia en la cultura participativa entre todos los agentes son un factor que agudiza las respuestas competitivas y excluyentes.

Dicho esto, es fundamental mencionar la oportunidad que se presenta con el cambio de modelo que se quiere impulsar, apostando por una mirada centrada en los cuidados, el desarrollo comunitario y la participación y la interseccionalidad. Esta respuesta ha sido validada por las personas que han participado en el proyecto. De esta manera, la construcción de espacios de diálogo permite ver, no sin dificultades, las potencialidades que hay para el apoyo mutuo, el encuentro y la solidaridad. En relación con estos principios, es importante mencionar los deseos propuestos para mejorar la vida en Zumarraga, que además de aquellas cuestiones ligadas a preocupaciones generales, como el empleo, la educación o la sanidad, pasan por el reforzamiento de lugares de encuentro, la puesta en marcha de cadenas de favores, de bancos del tiempo y de estrategias para el intercambio de recursos, tareas y saberes que activen el recurso comunitario para problemas de exclusión, marginalidad o soledad -junto a las respuestas institucionales-.

En este sentido, los espacios de diálogo permiten observar la priorización de principios que van encaminados a la justicia social a la hora de poner en 
marcha las propuestas 5 . El mayor consenso proviene de la creación de lazos, ya que se entiende como fundamental a la hora de conocer las realidades y necesidades de otras personas y grupos y, con ello, desvanecer estereotipos o fricciones. Existe consenso en la necesidad de impulsar iniciativas desde una apuesta intergeneracional, ya que se entiende como una oportunidad para promocionar el conocimiento entre diferentes vivencias. Y, así, mejorar la convivencia, fomentar la diversidad e impulsar la solidaridad, siendo una estrategia que puede contribuir a aliviar la soledad no deseada de algunas personas mayores y otros colectivos en situación de vulnerabilidad.

En los espacios participativos-deliberativos -en línea con las entrevistas - también se insiste en la importancia de revitalizar el tejido asociativo, haciendo mención a su papel promotor de la vida comunitaria y de la cohesión social ${ }^{6}$. Asimismo, hay consenso en la importancia en que las propuestas que se desarrollan ayuden a superar el conocimiento, superando estereotipos y desconfianzas entre la ciudadanía. Se asume que la diversidad representa una riqueza en sí misma y es necesario potenciarla y darle visibilidad. Además, es necesario contrarrestar estereotipos y rumores en el mismo nivel donde nacen -bien sea las redes sociales o las plazas-, así como realizar más actividades en asociaciones y colegios. Por ello, se insiste en el reforzamiento de lógicas interculturales en los proyectos que se desarrollen, que contribuyan al conocimiento mutuo y a la revalorización del mestizaje y la cohabitación de las distintas experiencias. A este respecto, la trayectoria del Ayuntamiento de Urretxu en proyectos de diversidad cultural puede ser un referente.

Una herramienta fundamental para encaminarse hacia estos principios es la participación ciudadana, a la que se atribuye un papel central en la puesta en marcha de otro modelo social basado en la horizontalidad y la corresponsabilidad. De esta forma, se justifica en su capacidad para empoderar a la ciudadanía, para visibilizar y legitimar la voz de los colectivos vulnerables y, con ello, fomentar la convivencia y romper con la exclusión y los prejuicios. Por ese motivo, es importante crear espacios físicos de encuentro para que la ciudadanía pueda dar a conocer sus propias necesidades y conocer las de las demás, así como rentabilizar los ya existentes.

Un recurso fundamental para ello son los barrios, que se señalan como fundamentales a la hora de construir alternativas para vertebrar en igualdad del municipio, pues para fomentar la participación es

5 En el taller de criterios, las personas participantes tienen que priorizar unos principios, facilitados por el equipo investigador a partir del análisis de las entrevistas, que consideren más relevantes para seleccionar las propuestas que posibiliten mejorar la situación del municipio en clave de cuidados comunitarios.

${ }^{6}$ Se recuerda la necesidad de fomentar la cooperación y no la competitividad no solo entre asociaciones, sino también entre los ayuntamientos de Urretxu y Zumarraga, que comparten un mismo núcleo urbano. imprescindible visibilizar y revitalizar las dinámicas del territorio, especialmente las parte más periféricas -escuchando y respondiendo a sus demandas según criterios de proximidad-. Por último, se indica la necesidad de potenciar la capacidad pedagógica de la participación y favorecer la socialización de valores como el cuidado, la dignidad, la solidaridad o el reconocimiento. Además, se recuerda que es necesario recuperar y fomentar las relaciones humanas reales. $Y$ una herramienta fundamental para encontrarse es el idioma. En un escenario menos euskaldun que en el entorno guipuzcoano se destaca la importancia de crear un vínculo saludable en torno al euskera y que esta lengua sea una herramienta integradora.

\subsection{Imaginando lo posible (desde esos saberes femeninos)}

Una vez que se ha reflexionado sobre los criterios que deben primar en los proyectos municipales, se construyen una serie de propuestas teniendo en cuenta las demandas obtenidas en el trabajo de campo y las referencias de otros territorios, pues la falta de experimentación social y la dificultad en el acceso mediático a prácticas transformadoras debilita la capacidad de imaginar otros modelos.

Una de las iniciativas sobre las que se trabaja son las huertas urbanas, pensadas como un "espacio donde se fomenta la biodiversidad. La vegetal, la animal y la humana. Es un lugar donde personas diferentes unen sus esfuerzos y su sabiduría para cultivar la vida comunitaria. La herramienta básica de trabajo es el respeto" (Sampietro et al., 2018: 101). De esta forma, un grupo de personas se organiza para reinventar cualquier recoveco de la ciudad - un parque, terreno libre alrededor de un árbol urbano, un solar o espacio descuidado, etc.- convirtiéndolo en un pequeño jardín y/o huerta, entre otros. En definitiva, pequeños lugares que pueden dar pie a ofrecer una segunda oportunidad a espacios degradados o condenados al asfalto a través de notas de color y de olor que simbolicen la vuelta a la naturaleza y a la revalorización de lo que, hasta hace no tanto, era el centro de vida, también en Zumarraga. Como explica Nerea Morán, arquitecta y urbanista del colectivo Surcos Urbanos:

La escala local es la más adecuada para acoger procesos de reapropiación del espacio y de creación de un paisaje urbano que responda a las necesidades e iniciativas de sus habitantes. Mediante la participación en las decisiones sobre el entorno, y en su misma configuración, los ciudadanos dotan a los espacios de identidad, expresan la diversidad social y cultural y, por tanto, el desarrollo de sentimientos de reconocimiento, apropiación y responsabilidad hacia el entorno.

Además, estos huertos ofrecen una oportunidad para intensificar las relaciones sociales. En este sentido, esta experiencia pensada desde la cogestión de 
espacios tiene el potencial para poner en relación diferentes generaciones y estilos de vida. Y, con ello, reivindicar, visibilizar y valorar el conocimiento de nuestros mayores a través de un proceso de descubrimiento y aprendizaje entre los más jóvenes, así como entre generaciones más ancladas en lo urbano. Se pueden destacar el Programa Huertos de Ocio de Vitoria-Gasteiz, la Red de Huertos Comunitarios de Barcelona o el Parque Miraflores de Sevilla.

Otra de las propuestas se dirige a la creación de un centro comunitario cogestionado, para generar una alternativa a los espacios institucionales que son un recurso destacado para actividades culturales y sociales. En este caso, se reflexiona sobre la construcción de un lugar que se convierta en el punto neurálgico de la dinamización de uno o varios barrios para avivar la relación entre los diferentes actores que conforman Zumarraga. Es decir, un pequeño (gran) laboratorio de cuidados como punto de encuentro desde el que potenciar redes y vínculos comunitarios a través de la formación, la realización de actividades culturales y deportivas, o el fomento del ocio y el tiempo libre. Por su larga trayectoria, destacan los Centros Comunitarios de Iniciativa Social de Pamplona que, dirigidos especialmente a la infancia y adolescencia, funcionan de forma cogestionada y son todo un referente de desarrollo comunitario en los barrios de la ciudad.

Por otro lado, la soledad no deseada ha sido otro tema recurrente. Aunque en el trabajo de campo se menciona particularmente a las mujeres mayores, en una sociedad cada vez más individualista no es difícil que cualquiera termine afectada por ella. Por ello, es necesario poner el foco en abordar una problemática que tiene su raíz en la desconexión social. Así, un proyecto de prevención de la soledad implica, por un lado, coordinar recursos públicos que en muchas ocasiones ya existen. Tan solo hace falta hacerlos llegar a las personas afectadas. Por ello, es necesario crear una red de observadores entre la ciudadanía identificando también personas clave como cuidadoras, enfermeras, farmacéuticas, médicas, etc., pero no solo. $Y$ activar las redes vecinales y los recursos públicos tanto en la identificación como en el acompañamiento, generando puntos amigos donde poder compartir. Es decir, para crear lugares de encuentro utilizando los servicios públicos - centros de salud, colegios, biblioteca, polideportivo, etc.-, comercio local -cafeterías, librerías, mercados...-, tejido social -asociaciones- 0 , incluso, diversos espacios comunes - bancos en parques, plazas-. Un ejemplo a este respecto es el programa de Prevención de la Soledad no Deseada dentro de Madrid, Ciudad de los Cuidados, o el Proyecto Radars de Barcelona. En ambos municipios, el ayuntamiento pone en marcha una experiencia para identificar y combatir situaciones de soledad no deseada y aislamiento social a través de una red de apoyo mutuo.

Además, también se reflexiona acerca del papel de las tecnologías de la información y la comunicación para potenciar los cuidados comunitarios en las sociedades actuales. En muchas ocasiones se mira con cierta desconfianza a los medios de comunicación por su capacidad de manipulación o a las redes sociales por su efecto deshumanizador en las relaciones personales. Bajo estas sombras, se olvida que, puestas en manos de la ciudadanía, también pueden tener un impacto positivo y funcionar como una herramienta fundamental a la hora de pensar en el desarrollo local. Por ello, no podíamos dejar pasar la oportunidad de plantear una propuesta en clave de construcción colectiva del conocimiento. Así, contar con un espacio de comunicación comunitaria se constituye como una oportunidad para la narración directa de vecinas y vecinos de Zumarraga que responda a sus propias demandas. Además, una experiencia en este campo puede ser fundamental si se implican aquellos grupos señalados como otros. Es decir, colectivos vulnerables cuya imagen, en muchas ocasiones, se construye a golpe de estereotipos y en los que recaen desconfianzas y hostilidades. Pues supone la oportunidad de mostrar sus puntos de vista y estilos de vida de primera mano con el fin de que la ciudadanía se (re)conozca. Y, en definitiva, puede ser una plataforma desde la que reforzar otros proyectos centrados en mostrar, visibilizar, valorizar y poner en relación las diferentes culturas, idiomas, realidades y trayectorias que cohabitan en Zumarraga desde una perspectiva inclusiva.

También es una oportunidad para la formación en diferentes materias que pueden servir para capacitar y empoderar a las personas participantes tanto de forma individual como colectiva. Por un lado, detenerse en el propio concepto de comunicación no violenta pensada desde la diversidad y el diálogo (Buraschi et al., 2017; Sclavi, 2003) que posibilite socializar en nuevos valores. Es decir, una comunicación que rehúya de dinámicas muy extendidas en nuestras sociedades basadas en la confrontación y el debate. De esta manera, la comunicación comunitaria permite una conexión en torno a la diversidad cultural así como intergeneracional, ya que juega con un instrumento que fascina a la parte más joven de la sociedad, mientras que puede verse como una oportunidad para formar a parte de la población tocada por la brecha digital.

Como ejemplo queremos hacer referencia a Teño unha horta en San Sadurniño (2015-) y Pasión de Palomares (2006). La primera se inserta dentro del programa Fálame de San Sadurniño, un espacio de capacitación de alfabetización digital y audiovisual en un marco de empoderamiento individual y colectivo. Se trata de un canal de Youtube que busca difundir el conocimiento y la experiencia de estas mujeres a través de una serie de videotutoriales en el que explican trucos y tareas tradicionales respecto al trabajo en el campo. La segunda está inserta en un proceso de planificación urbanística y económica en Palomares del Río (Sevilla). Se trata de una telenovela creada por la ciudadanía de esta localidad que se 
proyecta en bares, plazas y otros espacios comunes, así como en la televisión local. La producción narra las problemáticas del pueblo y busca fomentar la reflexión colectiva desde una campaña de ilusionismo social (Encina et al., 2007).

Todas estas propuestas se convierten en una oportunidad para experimentar en el ámbito comunitario, trabajando desde la justicia social en lugar de seguir fortaleciendo las lógicas competitivas y excluyentes. Así, aprovechando de nuevo las palabras de Silvia Navarro Pedreño: “En el páramo desolador del interés privado y la defensa propia, se multiplican hoy, como esperanzadores oasis sociales, múltiples iniciativas modestas y efímeras, locales y a un nivel micro, que son emprendimientos cooperativos ad hoc" (Navarro Pedreño, 2017: 250). Zumarraga se ha sumado a este entramado municipal que apuesta por contribuir al cambio social.

\section{Conclusiones: los cuidados comunitarios como respuesta creativa}

Las vulnerabilidades y necesidades que se detectan en el proceso iniciado en Zumarraga en 2017 no son exclusivas de este territorio y esta fotografía general es compartida en otros contextos. Por ello, es importante cartografiar la respuesta dada por la política local de este ayuntamiento que, al igual que otros muchos, está apostando por alternativas creativas que permitan abordar los dolores crecientes y fuertemente interconectados presentes en nuestras sociedades. Es decir, respuestas inclusivas a las consecuencias del modelo productivo actual y de sus constantes sacudidas, a los retos del envejecimiento de la pirámide poblacional, o de los movimientos migratorios. Y, con ello, respuestas inclusivas al individualismo y la precariedad, a la soledad, a la otredad, los prejuicios y el miedo.

En este sentido, a partir de las investigaciones diagnósticas y propositivas realizadas en los dos últimos años, ha incorporado los cuidados comunitarios como lógica de trabajo futuro, generando alternativas optimistas capaces de transformar el escenario. A este respecto, se ha aprobado recientemente la ordenanza para poner en marcha las huertas urbanas y se están dando los primeros pasos para iniciar un proceso de relatorías, que sirva como experimentación social en el reforzamiento de los lazos vecinales. Una iniciativa que contribuya también a la revalorización de vivencias otras que han pasado desapercibidas para una historia escrita en clave de dominación - patriarcal, clasista o colonial, entre otras muchas-.

Todas estas iniciativas $-\mathrm{y}$ otras muchas que suceden en lo micro- tienen que ser constantes pues es importante visibilizar otras formas de hacer política, que exigen tiempo, cercanía y mimo. Unas formas que no se basan en la exclusión de otras personas -especialmente las más vulnerables- sino en articular espacios que nos permitan conocernos, encontrarnos y reforzarnos como comunidad. Cuando muchas veces los gobiernos locales parecen ser presentados como entes pasivos y meros gestores de servicios, lo cierto es que tienen un rol fundamental en la decisión de democratizar - o no- la política, constituyéndose en un enclave estratégico para la transformación social. Reivindiquemos su papel como tal. 


\section{Bibliografía referenciada}

AGUADO-PELÁEZ, D. y MARTÍNEZ-GARCÍA, P. (2019): “¿Quién sostiene la vida? Análisis interseccional de las cuidadoras remuneradas de Urretxu y Zumarraga" [estudio inédito resultado de una beca de investigación financiada por la Dirección de Igualdad de la Diputación Foral de Gipuzkoa].

AGUADO-PELÁEZ, D. y MARTÍNEZ-GARCÍA, P. (ARADIA COOPERATIVA) (2018): Construyendo propuestas hacia un modelo sustentado en los cuidados comunitarios, Zumarraga, Área de Servicios Sociales, Igualdad y Diversidad.

AHEDO, I. (2018): Diagnóstico de fortalezas y debilidades para la participación entre el cuerpo político y técnico, Zumarraga, Área de Administración Local y Participación Ciudadana.

AHEDO, I.; ARANDA, U.; y URETA, M. (2018): Diagnóstico de Diversidad y Vulnerabilidad en Zumarraga, Zumarraga, Área de Servicios Sociales, Igualdad y Diversidad, «http://www.zumarraga.eus/ es/-/informe-ejecutivo-del-diagnostico-dediversidad-y-vulnerabilidad-en-zumarraga-2018?inheritRedirect=true\&redirect $=\% 2 \mathrm{Fes} \% 2 \mathrm{~F}$.

ARRIETA, F. et al. (2018): “La comunidad al rescate: algunas reflexiones sobre las políticas estatales de activación comunitaria en Europa", Zerbitzuan, 65, págs. 5-18, 〈https://doi.org/10.5569/11347147.65.01>.

BURASCHI, D.; AMORAGA MONTESINOS, F.; y OLDANO, N. (2017): "Diálogo y transformación en los procesos participativos: la experiencia de los laboratorios dialógicos en las Islas Canarias", Rivista di Pedagogia Critica, no1, 〈http://www. educazioneaperta.it/>.

CARRASCO, C. (2013): "El cuidado como eje vertebrador de una nueva economía”, Cuaderno de Relaciones Laborales, vol. 31, no 1, págs. 39-56, «http://
revistas.ucm.es/index.php/CRLA/article/ view/41627>

COLLINS, P. HILL (1990): Black Feminist Thought. Knowledge, counsciousness and the politics of empowerment, London, Routledge.

COLLINS, P. HILL (2017): “The difference that power makes", Investigaciones feministas, vol. 1, nํㅛ, págs. 19-39, 〈https://revistas.ucm.es/index.php/ INFE/article/view/54888/51188>.

COMAS, D. (2014): "Los cuidados y sus máscaras. Retos para la antropología feminista", Mora, no 20, págs. 167-182, <http://revistascientificas.filo. uba.ar/index.php/mora/article/view/2339〉.

ENCINA, J. et al. (2007): La ciudad a escala humana, Palomares del Río, Universidad Libre para la Construcción Colectiva (UNILCO) y Atrapasueños.

FRASER, N. (2008): Scales of Justice. Reimagining political space in a globalizing world, Cambridge, Polity Press.

- (2015): Fortunas del feminismo, Madrid, Traficantes de Sueños.

FUNDACIÓN MATIA (2015): Diagnóstico de Amigabilidad de Zumarraga / Diagnóstico Zumarraga Lagunkoia, Zumarraga, Área de Servicios Sociales, Igualdad y Diversidad, «https://www. euskadilagunkoia.net/es/component/content/ article id=334: presentacion-del-diagnosticode-amigabilidad-en-zumarraga\&ltemid $=367$ > .

GIMÉNEZ, S.; CORTÉS, Ma C.; y SÁEZ, J. (2016): Discriminación y comunidad gitana. Informe anual FSG 2016. Número especial sobre antigitanismo, Madrid: Fundación Secretariado Gitano, 〈https://www.gitanos.org/ centro_documentacion/herramientas/cajas/ antigitanismo.html.es〉. 
HERNANDO, A. (2012): La fantasía de la individualidad. Sobre la construcción sociohistórica del sujeto moderno, Buenos Aires, Katz Editores.

HERRERO, Y.; PASCUAL, M.; y GONZÁLEZ REYES, M. (2018): La vida en el centro. Voces y relatos ecofeministas, Madrid, Libros en Acción.

JULIANO, D. (2017): Tomar la palabra. Mujeres, discursos y silencios, Barcelona, Ediciones Bellatera.

JUNCO, C.; PÉREZ OROZCO, A.; y DEL RÍO, S. (2004): “Hacia un derecho universal de cuidadanía (sí, de cuidadanía)", 〈https://www.lahaine.org/mm ss_est_esp.php/hacia-un-derecho-universaldè.

MARÇAL, K. (2016): ¿Quién le hacía la cena a Adam Smith? Una historia de las mujeres y la economía, Barcelona, Debate.

MARTÍNEZ-BUJÁN, R. (2014): "Los modelos territoriales de organización social del cuidado a personas mayores en los hogares", Revista Española de Investigaciones Sociológicas, $\mathrm{n}-145$, págs. 99-126, 〈http://dx.doi.org/10.5477/cis/ reis.145.99>.

MARTÍNEZ-PALACIOS, J. y MARTÍNEZ-GARCÍA, P. (2017): "Justicia social, interseccionalidad y profundización democrática”, Investigaciones feministas, vol. 1, no 8, «https://revistas.ucm. es/index.php/INFE/issue/view/3103/showToc>.

MEDINA BRAVO, P. y RODRÍGUEZ ALSINA, M. (2005): "Las emociones como barreras y accesos a la diversidad cultural", Redes.com, Revista de Estudios para el Desarrollo Social de la Comunicación, no 2, págs. 19-28, «http:// revista-redes.hospedagemdesites.ws/index. $\mathrm{php} /$ revista-redes/article/view/49/43〉.
MORÁN, N. (2010): “Agricultura Urbana: un aporte a la rehabilitación integral”, Papeles de relaciones ecosociales y cambio global, n- 111 , págs. 99-111, «http://oa.upm.es/12160/1/INVE MEM_2010_76416.pdf .

NAVARRO PEDREÑO, S. (2017): Saber femenino, vida y acción social. Dar a luz experiencias creadoras, Madrid, Editorial CCS.

PÉREZ OROZCO, A. (2014): Subversión feminista de la economía. Aportes para un debate sobre el conflicto capital-vida, Madrid, Traficantes de Sueños.

- (2011): "Crisis multidimensional y sostenibilidad de la vida", Investigaciones feministas, vol. 1, págs. 29-53, 〈http://dx.doi.org/10.5209/ rev_INFE.2011.v2.38603〉.

PRADA SANTAMARÍA, A. (2016): Los Zumarragarras. Una búsqueda de nuestra identidad a través de los padrones de habitantes (1877-2015), Zumarraga, Ayuntamiento de Zumarraga.

SAMPIETRO, P. et al. (2018): La ciudad comestible. La Naturaleza ha decidido volver a la ciudad, Barcelona, Editorial Morsa.

SANTOS, E. et al. (2018): Metodologías participativas y democracias transformadoras, Madrid, Red Cimas, Colección Construyendo Ciudadanía/15.

VV. AA. (2006): Pasión de Palomares [telenovela participativa], 〈https://vimeo.com/31080405〉.

VV. AA. (2015-): Teño unha horta en San Sadurniño [canal de Youtube], «https://www.youtube.com/channel/ UCgYziuQoVFEOoXRjhZyggNQ〉.

VILLASANTE, T. (2017): Democracias transformadoras. Experiencias emergentes y alternativas desde los comunes, Madrid, El Viejo Topo. 